\title{
SCRIPT FILES APPROACH IN THE POWER QUALITY EVENTS GENERATION
}

\author{
$U D C(((004.9: 621.317 .347)+621.395 .38): 621.8 .037)$
}

\section{Dragan Živanović ${ }^{1}$, Milan Simić ${ }^{1}$, Dragan Denić ${ }^{1}$, Živko Kokolanski ${ }^{2}$ \\ ${ }^{1}$ University of Niš, Faculty of Electronic Engineering, Niš, Republic of Serbia ${ }^{2}$ Ss. Cyril and Methodius University, Faculty of Electrical Engineering and Information Technologies, Skopje, Macedonia}

\begin{abstract}
Generation of typical power quality $(P Q)$ events, based on Script files and virtual instrumentation, is presented in this paper. Such approach provides the definition and generation of three-phase voltage signals with various $P Q$ events defined according to relevant international quality standards. Using of Script files enables an easy and flexible generation of long-time voltage signals with complex $P Q$ disturbances, according to the predefined test algorithms and scenarios. Detailed front panels and block diagrams of developed virtual instrument for signal generation are described in the paper. As specific examples, generated signals defined using the Script files are presented. An experimental confirmation of the described software supported method is performed using the three-phase $P Q$ analyzer Fluke 435. Some specific test waveforms and obtained experimental results are shown.
\end{abstract}

Key words: virtual instrumentation, signal generation, power quality events, script files,

\section{INTRODUCTION}

Various problems and signal disturbances in one-phase or three-phase electrical power distribution networks can be reasons for possible degradation of the electrical power quality (PQ) level. The optimal quality level of electrical power delivered to the final customers is defined according to the relevant international quality standards and regulations $[1,2]$. For example, the PQ standard EN 50160 defines a nominal reference and acceptable tolerances of typical quality parameters in public electrical power distribution systems, under normal operating conditions. Various sophisticated microprocessor supported instruments and measurement systems for continuous monitoring and analysis of electrical power distribution and consumption are developed in recent years. Software supported instruments and

Received September 11, 2018

Corresponding author: Dragan Živanović

University of Niš, Faculty of Electronic Engineering, Aleksandra Medvedeva 14, 18106 Niš, Republic of Serbia

E-mail: dragan.zivanovic@elfak.ni.ac.rs 
equipment can be very useful and successful in the design and realization of computer-based PQ measurement applications [3-7]. Instruments applied for measurement and analysis of standard PQ parameters and possible network disturbances must be previously tested and verified in order to fulfill the required metrological performances and characteristics.

The software supported approach, presented in this paper, enables generation of the reference signals suitable for testing typical instruments for detection and measurement of standard PQ events and disturbances. This software supported solution for providing predefined long-time test signals is based on virtual instrumentation software, and the implementation of Script files. An approach enables generation of complex sequences according to the predefined test scenarios, including various PQ events defined by quality standard EN 50160.The developed solution is experimentally verified by the testing the professional instrument for PQ events detection - three phase analyzer Fluke 435 [8]. The basic software configuration of the signal generator, and experimental test procedure are shown and described in the paper.

\section{DESCRIPTION OF SOFTWARE SUPPORTED PQ EVENTS GENERATION}

The software supported generation of PQ events and standard disturbances is implemented using virtual instrumentation concept, with LabVIEW application software, acquisition card NI PCIe 6343, and additional power amplifier, in order to provide the nominal RMS output signal value of $230 \mathrm{~V}$. Real-time signal generation is enabled using analog output channels of the acquisition card NI 6343. This is a 32-channel D/A acquisition card, with maximum output range of $\pm 10 \mathrm{~V}$ and 16-bit resolution [9].

This solution is experimentally confirmed by testing the measurement instrument - PQ analyzer Fluke 435 Series II. The block configuration of the experimental test system, including a computer with LabVIEW virtual instrumentation software, data acquisition card NI 6343 with connector block SCB-68A, and external PQ signal amplifier, is presented in Fig. 1.

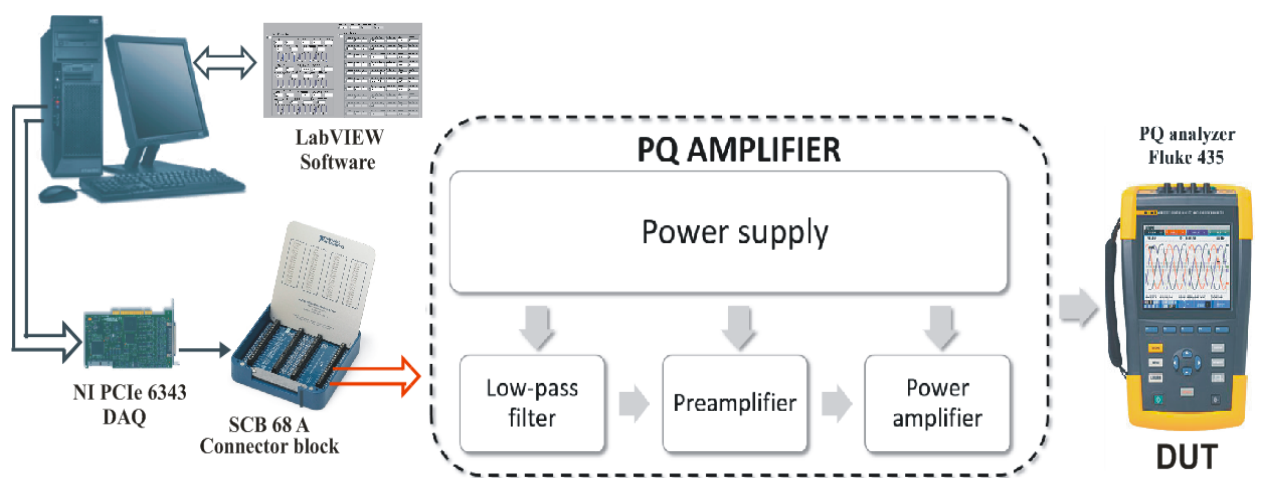

Fig. 1 Block diagram of experimental system for testing quality analyzer Fluke 435 using LabVIEW based PQ events generator 
In order to provide the reference output level required for testing instruments for PQ measurement, the signals generated at the acquisition card analog outputs must be amplified to the nominal power line voltage level of $230 \mathrm{~V}$. This specific signal amplifier is developed using several processing segments: a low pass antialiasing filter to restrict signal bandwidth and eliminate noise, a preamplifier to amplify the input signal to a required reference level and a power amplifier to amplify the input signal to the nominal voltage level of $230 \mathrm{~V}$. The detailed realization of signal amplifier is described in the previously published paper [10].

Generally, this solution enables a simulation of three-phase voltage waveforms in order to simulate a three-phase power distribution network.

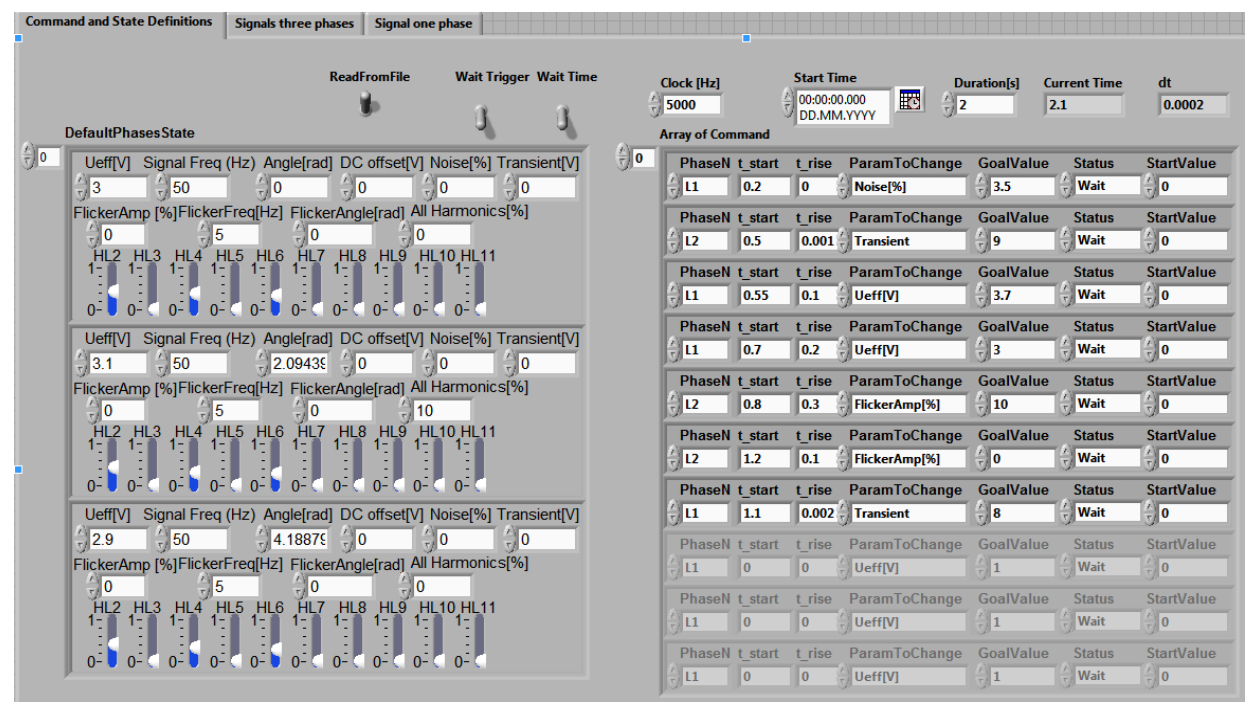

Fig. 2 Front panel of LabVIEW PQ signal virtual instrument

The virtual instrument includes two basic functional segments: a graphical user interface (front panel), and an executive software code (block diagram). The basic front panel of the developed virtual instrument enables selecting and varying of the default signal parameters, using the number of control functions and knobs, implemented in the front panels and block diagrams of the developed software application. The control front panel of LabVIEW based virtual instrument for PQ events selection and generation is presented in Fig. 2. At the left side of this front panel are shown default reference values of signal parameters per each of three phases of generated signals: nominal signal amplitude and frequency values, phase angle, signal DC offset percentage noise level, flicker amplitude, and flicker frequency values, including overall adjustment of high-order signal harmonics. Using the array of the control knobs for harmonics selection, the maximum percentage level of the specific high-order harmonics can be precisely defined. Based on the defined parameters, the virtual instrument successively determines samples for signal generation and sends specific values to the data acquisition card buffer for the generation of output signals. Also, the presented front panel includes an array of 
commands for changing signal parameters in the defined time, including the defined rising time, and goal parameter values. This specific array for changing parameters, shown on the right side of the front panel in Fig. 2, enables a generation procedure with complex predefined test scenarios and algorithms.

Besides definition of default parameter values for signal generation, in the control front panel of the virtual instrument is implemented a special function called "read from file", which provides the reading of predefined test waveforms, previously recorded in files using *.txt data format. This approach with script file definition of test voltage waveforms enables an easy repetition of various test algorithms, automated generation of test files and manual editing or combining various test scenarios by using some standard text editors.

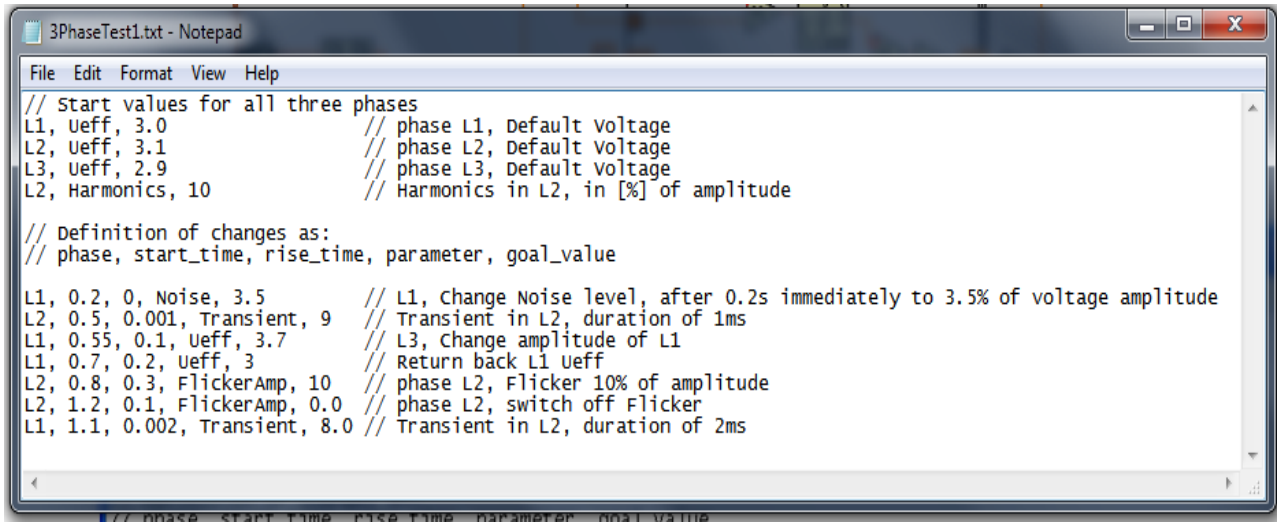

Fig. 3 Example of test voltage waveform with various signal disturbances defined in .txt script file

A specific example of the test signal defined in the script file, including changing of default parameter at the beginning, and then array of commands to change particular parameters, is shown in Fig. 3. One command is based on one line of text. Comments can be included anywhere after '//' characters. The line, which changes one of the default values, includes three parameters separated with a comma ',' character. The line starts with the name of the voltage phase, followed by the name of the parameter. Goal values of specific signal parameters are included at the end of the line.

It is possible to put a very large number of parameter and level of disturbances changing action, for any of three phases, enabling very complex and non-repetitive testing scenarios, where modern power quality measurement device will record the exact time of particular disturbances. During the development of such instruments, the complex scenario which can be loaded from the file, and repeated for a different realization of the instrument itself, presents a useful tool. 


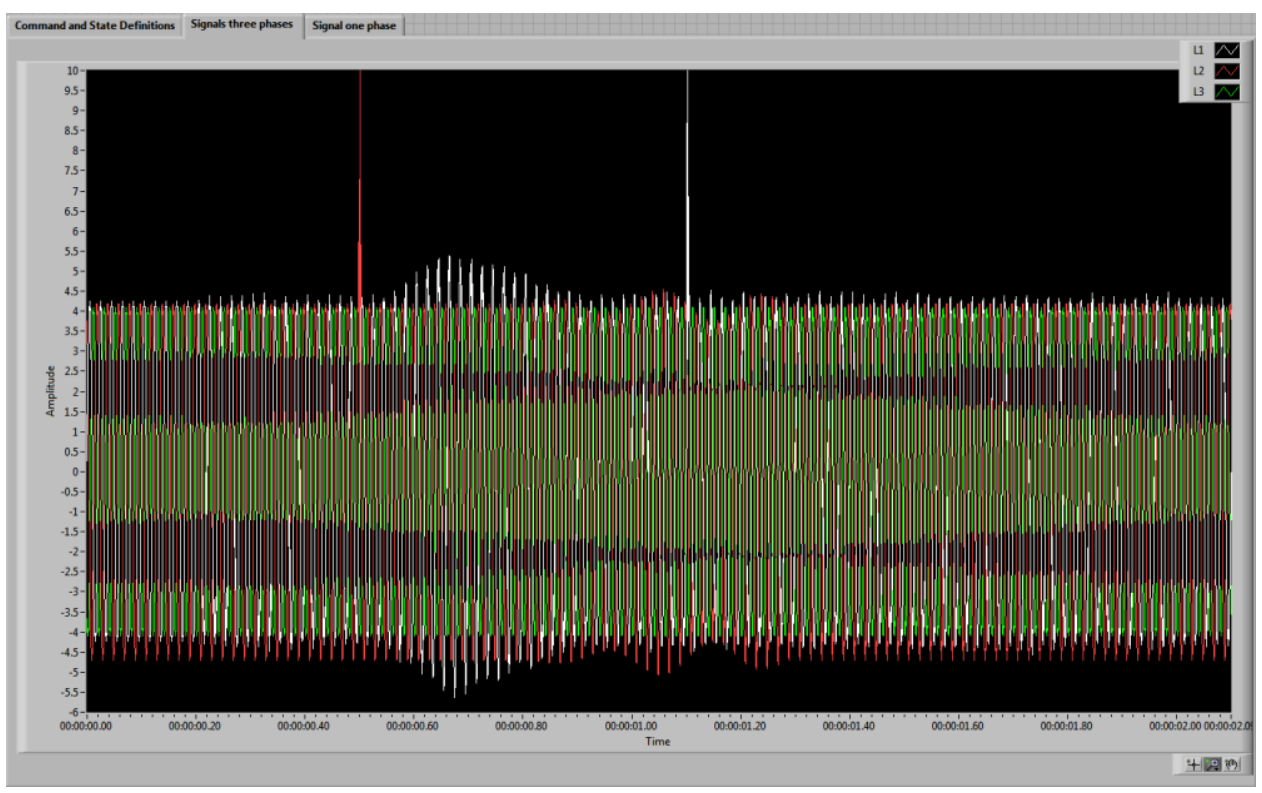

Fig. 4 Graphical presentation of three-phase voltage signal with disturbances

At Fig. 4. one can see all three phases generated based on the script file at Fig. 3. This is a short test scenario (two seconds) including transient per two phases, changing of noise level, and the amplitude of the first phase, and starting of flicker in the second phase. Exact time, duration, and the level of the mentioned steps can be easily seen in the script file itself, or at the front panel, after reading such file, presented at Fig. 2.

\section{BLOCK DIAGRAM OF PQ GENERATOR VIRTUAL INSTRUMENT}

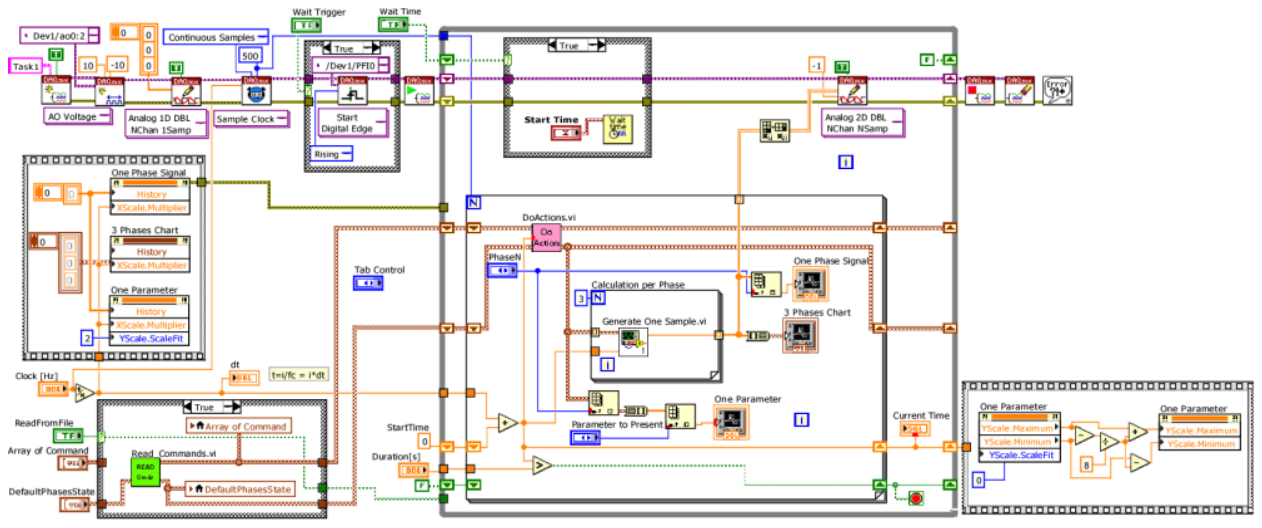

Fig. 5 Block diagram of LabVIEW virtual instrument 
A block diagram of a virtual disturbance generator can be seen in Fig. 5. Within the main program, the initiation of the hardware DAQ card is done. If synchronization with external instruments is required via the trigger signal, the digital trigger input is also enabled. At the same time, the script file that specifies the list of disturbances is loaded. Fig. 6 presents a block diagram of the subVI "Read_Commands.vi" that selects and loads the script file. Based on the loaded commands, the program changes the default values of the generator parameters, then creates an array of the signals disturbances. Each disturbance is defined by the time of the start, and the time for which the disturbance goes from 0 to the final amount (rise time and goal value).

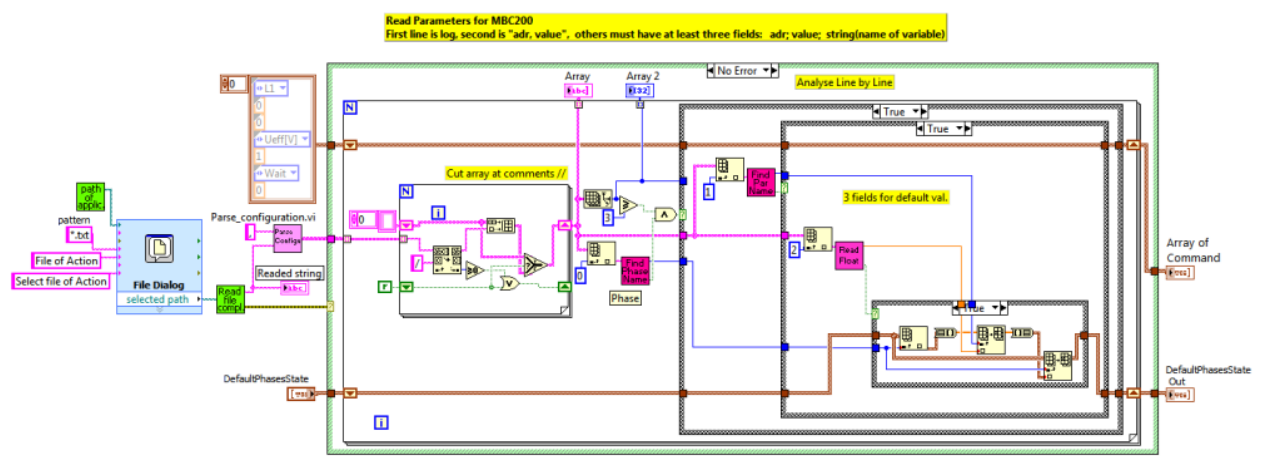

Fig. 6 Block diagram of "Read_Commands.vi"

In the main program (Fig. 5), a start time of the signal generation is then waiting. Signals are generated in a group of 500 samples (external for loop), which are simultaneously sent to the DAQ card buffer. Based on the defined clock, those 500 samples are generated for all three phases simultaneously. Before sending to DAQ cards, the samples are calculated on the basis of the current values of the signal parameter (amplitude, phase, disturbance, noise level, etc.).

The current phase of the signal is obtained based on the time elapsed since the start of the generation. That elapsed time is based on the current number of samples generated, and the defined sample rate of the generator. Disturbances, that is, the changes of the signal parameters, are obtained by interpreting the array of the disturbances, read from the script file.

The list of disturbances is interpreted in subVI "DoActions.vi", Fig. 7. In the loop of all disturbances that have not already been activated, the current time from the start of generation is compared with the specified time of the disturbance, and then the amount of change of the selected signal parameter is updated (based on current time, t_start, and t_rise), and finally the selected parameter is changed. 


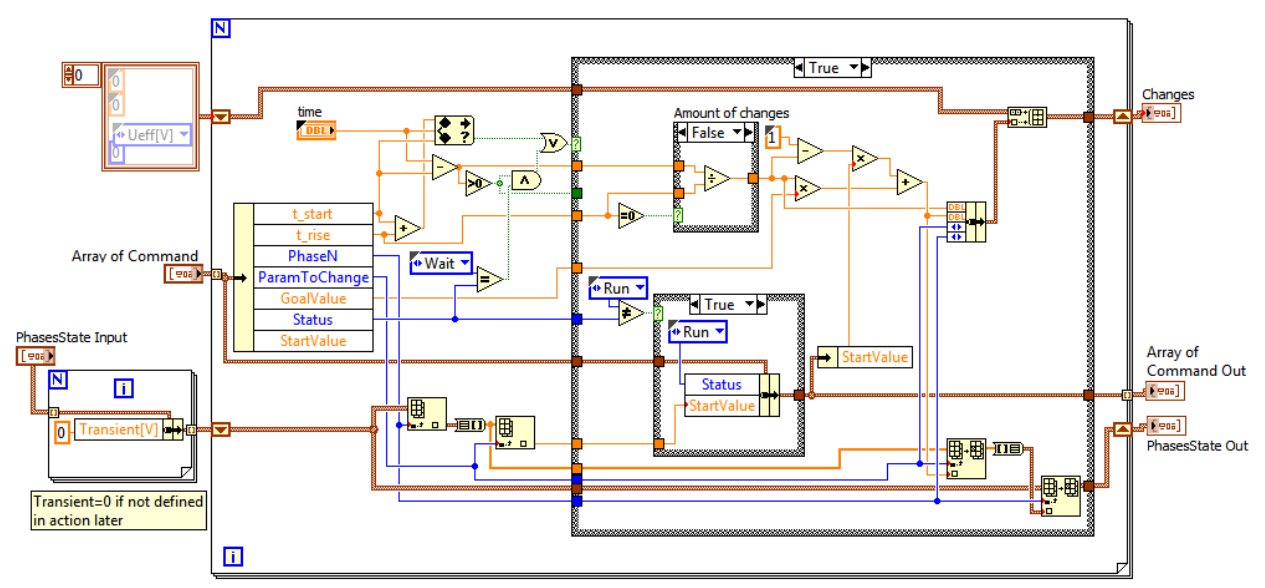

Fig. 7 Block diagram of "DoActions.vi"

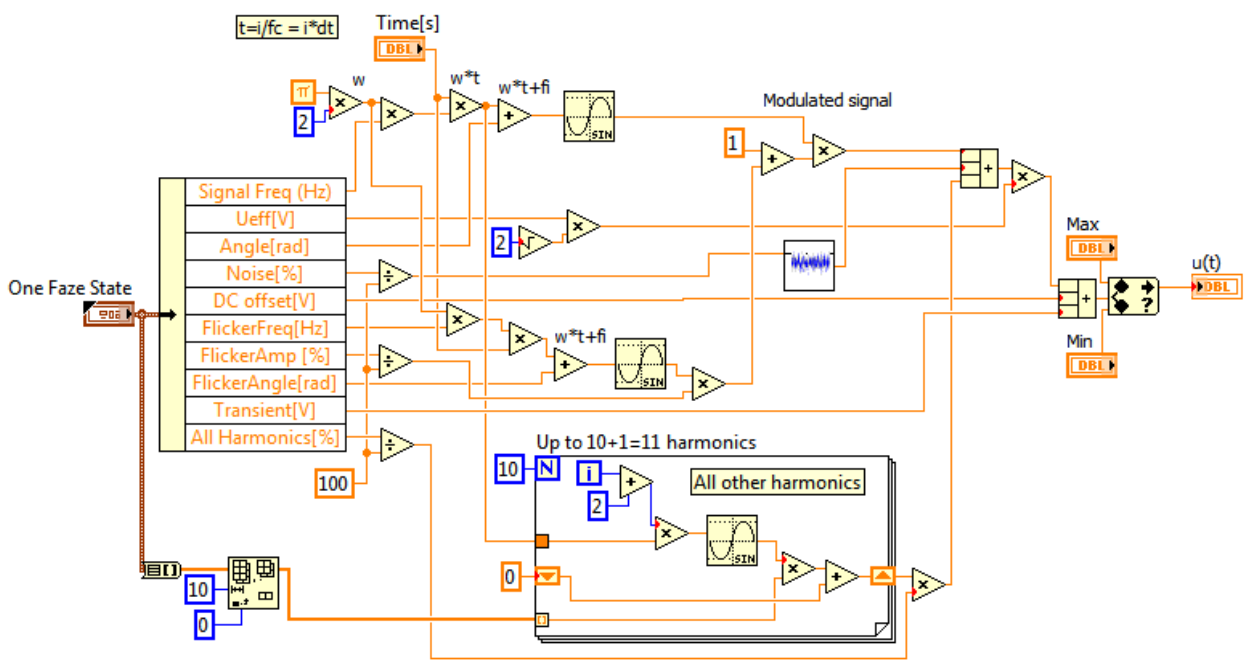

Fig. 8 Block diagram of "Generate One Sample.vi"

In the internal for loop of the main program, subVI "Generate One Sample.vi" is called for all three phases. It calculates the current amplitude of the samples, Fig. 8. Within this procedure, the noise is added, the current value of all harmonics, the value of transitions, and flickers are defined, and then based on the current signal parameters, amplitude, phase, frequency and DC component, the current amplitude value are reached. Calculated samples in the groups of 500, for all three phases, are sent to the DAQ card, as already mentioned. At the same time, the graphics on the front panel are refreshed with the generated samples and current parameter values. 
As DAQ card has the large buffer for samples, it is not very hard to select PC computer capable enough to calculate samples in the real time. After that, the generation stability depends only on the clock frequency stability of the DAQ card, which is $50 \mathrm{ppm}$. It can be calculated that the cumulative time error of disturbances generation, after one hour of operation is about $18 \mathrm{~ms}$.

\section{SOFTWARE ANALYSIS OF EXPERIMENTAL RESULTS}

The block configuration of the experimental system for testing the three-phase PQ analyzer Fluke 435 is already presented in Fig. 1. Amplified voltage waveforms, generated by LabVIEW based signal generator with various disturbances, are sent directly to the voltage inputs of a device under test (DUT) - measurement instrument Fluke 435. Setup of the experimental process includes various tests with the generation of reference voltage signals with some typical variations of standard PQ disturbances. Testing of the instrument Fluke 435 is focused on detection of standard PQ disturbances: voltage swells, voltage dips, voltage transients, and voltage interruptions, in accordance with the recommendations of the European quality standard EN 50160. The communication between the tested instrument Fluke 435 and the control computer is provided using the standard Universal Serial Bus (USB) communication interface. The obtained measurement results and logged signal waveforms can be easily transferred to the computer, chronologically recorded, and accordingly processed.

The instrument Fluke 435 is set to operate in the Data logging mode. The time interval for each measurement cycle in the instrument data logger is adjusted to the minimum value of $0.25 \mathrm{~s}$. The duration of data logging is equal to the length of reference signals obtained from the generator. The logged waveforms and experimental results recorded in the instrument database are sent to the computer using USB communication interface. The sophisticated Power Log application software provides various possibilities for software analysis of recorded data and logged signals. In the paper will be presented some reports about measured and detected PQ disturbances (voltage swells, dips, rapid voltage changes, transients, interruptions, etc.), obtained by tested quality analyzer Fluke 435. The time diagram of measured RMS voltage values for $1800 \mathrm{~s}$ (30 minutes) long voltage test waveform, including a large number of standard disturbances, recorded using the Power Log application software, is shown in Fig. 9.

In this diagram are chronologically and clearly indicated the detected PQ disturbances with specific measured RMS voltage values related to each of the detected events. The script file including such a large number of various signal disturbances is generated using the additional LabVIEW application software for random generation of various signal disturbances. As this specific script file is recorded in the *.txt text file, it is possible to inspect, manually modify and store this file in the database of test scenarios, for repetition or later applications. 


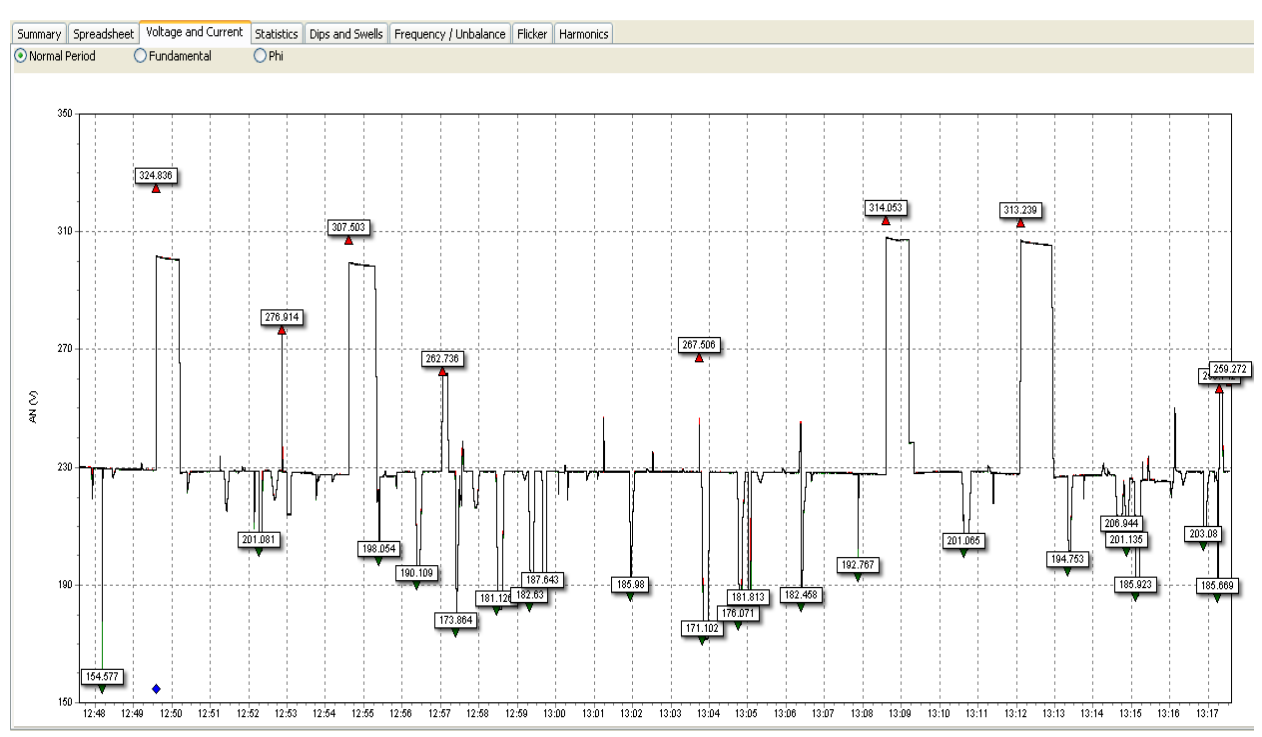

Fig. 9 Time diagram of measured RMS voltage values - 30 minutes long test signal with a large number of voltage disturbances

The instrument Fluke 435 detects quality disturbances according to the standard EN 50160 [1]. This standard defines acceptable limits of a nominal RMS voltage of $230 \mathrm{~V} \pm$ $10 \%(207 \mathrm{~V}$ to $253 \mathrm{~V})$. Voltage values larger than $253 \mathrm{~V}$ the instrument detects as the swell (SWL), while voltage values smaller than $207 \mathrm{~V}$ the instrument detects as the voltage dip (DIP). The instrument detects voltage interruption (INT) in the cases when the measured voltage values are smaller than $1 \%$ of the nominal RMS value $(1 \%$ of $230 \mathrm{~V})$. This practically means that interruptions will be detected for the voltage values smaller than $2.3 \mathrm{~V}$. For the voltage levels smaller than $10 \%$ of the nominal RMS value $(10 \%$ of $230 \mathrm{~V})$ the instrument indicates the rapid voltage change (CHG). For short-time and very fast rapid voltage rise, in an amount greater than $100 \mathrm{~V}$ in relation to the nominal voltage value, the instrument will detect a presence of voltage transient (TRA) [8].

As an example for the detection of specific PQ events and disturbances, in Fig. 10. is presented a detailed summary of chronologically detected disturbances in the test signal, recorded directly from the graphical display of the quality analyzer Fluke 435. This summary is obtained for 30 minutes long voltage test waveform generated with a large number of disturbances. This report gives just a small part of all detected signal disturbances, due to the space limitations in instrument graphical display. In instruments report summary are included following data: types of detected quality problems, exact dates and times for individual detections, voltage amount, and time duration for detected disturbances. 


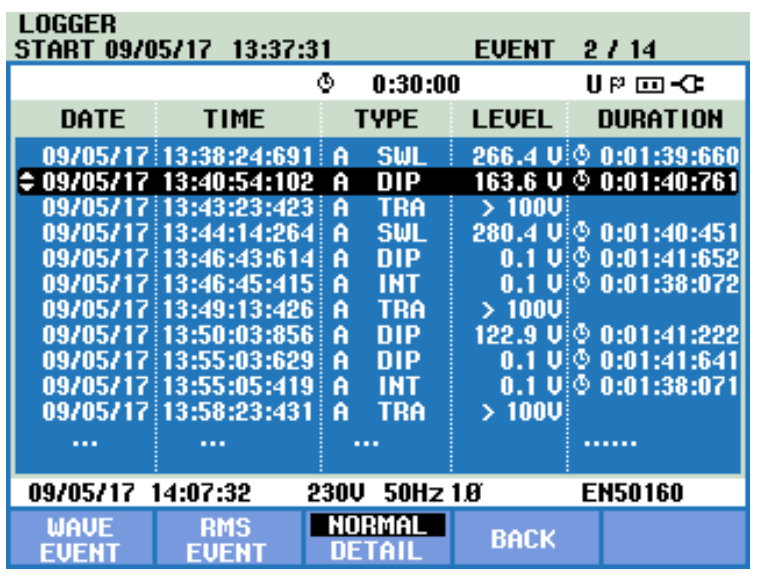

Fig. 10 Summary of detected PQ events recorded from graphical display of instrument Fluke 435 - 30 minutes test signal with large number of disturbances

\section{CONCLUSION}

A software-based generator of voltage signals with variations of standard PQ events, typical for real power distribution networks, is described in this paper. The procedure for signal generation is based on script files approach and virtual instrumentation concept. This generator enables easy and flexible repeatable testing of instruments and equipment designed for measurement and analysis of standard PQ parameters. Such an approach enables generation of specific test signals according to predefined complex scenarios, including typical PQ events defined according to the European quality standard EN 50160. For experimental confirmation of the developed signal generator, the procedure for testing instrument for detection of standard quality disturbances - the three-phase analyzer Fluke 435 is presented in the paper. The described test procedure is focused on the detection of standard quality disturbances using the instrument Fluke 435 for longtime test waveforms, generated with a large number of various PQ events and signal disturbances.

\section{REFERENCES}

[1] Copper Development Association, "Power quality application guide, Voltage disturbances, Power Quality PQ Standard EN 50160," 2004.

[2] A. Ferrero, "Measuring electric power quality: Problems and perspectives," Measurement, Elsevier, vol. 41, no. 2, pp. 121-129, 2008. [Online]. Available: https://doi.org/10.1016/j.measurement.2006.03.004.

[3] J. G. de la Rosaa, A. A. Péreza, J. C. Salasa, J. M. Fernándeza, A. M. Muñoza, "A novel virtual instrument for power quality surveillance based in higher-order statistics and case-based reasoning," Measurement, Elsevier, vol. 45, no. 7, pp. 1824-1835, 2012. [Online]. Available:

[4] https://doi.org/10.1016/j.measurement.2012.03.036.

[5] D. Montenegro, M. E. Hernandez, G. A. Ramos, "A realistic generator of power quality disturbances for practicing in courses of electrical engineering, ” Computer Applications in Engineering Education, vol. 23, no. 3, pp. 391-402, 2014. [Online]. Available: https://doi.org/10.1002/cae.21609. 
[6] Y. Lim, H.M. Kim, S. Kang, "A design of wireless sensor networks for a power quality monitoring system,” Sensors, vol. 10, no. 11, pp. 9712-9725, november 2010. [Online]. Available:

[7] https://www.ncbi.nlm.nih.gov/pubmed/22163436.

[8] C. Chunling, Q. Huihui, Z. Wei, W. Pengfei, "Transient power quality signal generator and detector platform," Energy Procedia, vol. 16 B, pp. 1380-1385, 2012. [Online]. Available:

[9] https://doi.org/10.1016/j.egypro.2012.01.219.

[10] V. Kumar, M. Kaur, "Power quality event generation in MATLAB/Simulink environment," International Journal of Advanced Research in Electrical, Electronics and Instrumentation Engineering, vol. 4, no. 7 , pp. 6055-6059. [Online]. Available: http://www.ijareeie.com/upload/2015/july/49_Power.pdf.

[11] Fluke Corporation, "Fluke 435 Series II, Three-phase energy and power quality analyzer,” Manual, 2012

[12] National Instruments Corporation, "PCIe NI 6343 multifunction data acquisition card,“ Specifications, Rev. 2014. [Online]. Available: http://www.ni.com/datasheet/pdf/en/ds-152.

[13] M. Simić, Z. Kokolanski, D. Denić, V. Dimcev, D. Živanović, D. Taskovski, "Design and evaluation of computer-based electrical power quality signal generator,” Measurement, Elsevier, vol. 107, pp. 77-88, 2017. [Online]. Available: https://doi.org/10.1016/j.measurement.2017.05.010. 